PROCEEDINGS OF THE

AMERICAN MATHEMATICAL SOCIETY

Volume 129, Number 1, Pages 181-190

S 0002-9939(00)05884-6

Article electronically published on August 17, 2000

\title{
BLOCK DIAGONALIZATION IN BANACH ALGEBRAS
}

\author{
ROBIN HARTE
}

(Communicated by David R. Larson)

\begin{abstract}
Reduction" of linear operators is effected by commuting projections; the spectrum of the operator is then the union of the spectra of its range and null space restrictions. Disjointness of these partial spectra implies that the projection "double commutes" with the operator, which in turn can be recognised as a curious kind of "exactness". Variants of this exactness correspond to various kinds of disjointness between the partial spectra.
\end{abstract}

\section{INTRODUCTION}

"Reduction" of an operator $T$ on a linear space $X$ means writing $X=X_{0} \oplus X_{1}$ as the direct sum of a pair of complementary invariant subspaces $T X_{j} \subseteq X_{j}$ for $T$ : the philosophy is that each of the induced operators $T_{j}$ is in some sense simpler than the original operator $T$, whose behaviour on $X$ can be reconstructed from the behaviour of $T_{0}$ and $T_{1}$. When $X$ is a Banach space and $T$ is continuous it is natural to ask that the subspaces $X_{j}$ be closed also; then the open mapping theorem says that there is a bounded projection

$$
Q=Q^{2} \in B L(X, X) \text { with } Q T=T Q,
$$

commuting with $T$, whose range and null space furnish the subspaces

$$
X_{1}=Q(X) \text { and } X_{0}=Q^{-1}(0) \text {. }
$$

In this situation the behaviour of $T$ is rather easily recovered from $T_{1}$ and $T_{0}$; for example the spectrum

$$
\sigma(T)=\sigma\left(T_{1}\right) \cup \sigma\left(T_{0}\right)
$$

Of course it is not at all clear that such reduction can be achieved in a non-trivial way: the operator $T$ may have no invariant subspaces other than $\{0\}$ and $X$, such invariant subspaces may not have closed complements, and if they are complemented, they may not have invariant complements. One situation in which such a projection certainly can be found is when the spectrum of $T$ can be written as a disjoint union of closed subsets

$$
\sigma(T)=K_{1} \cup K_{0} \text { with } K_{1} \cap K_{0}=\emptyset .
$$

Received by the editors December 15, 1997 and, in revised form, March 10, 1998, October 6, 1998, and March 31, 1999.

1991 Mathematics Subject Classification. Primary 47A13; Secondary 15A21, 15A18.

Key words and phrases. Commuting idempotent, double commutant, spectral disjointness, exactness conditions. 
A projection $Q$ satisfying (0.1) is given by a Cauchy integral

$$
Q=\chi_{K_{1}}(T)=\frac{1}{2 \pi i} \oint_{K_{1}}(z I-T)^{-1} d z .
$$

Here the disjoint subsets of the spectrum coincide with the spectra of the restricted operators

$$
K_{1}=\sigma\left(T_{1}\right) \text { and } K_{0}=\sigma\left(T_{0}\right) .
$$

Notice in particular that the reduced operators $T_{j}$ induced on the invariant subspaces have mutually disjoint spectra. This need not always happen: in a provocative observation Koliha 8] has noticed that in finite dimensions it is necessary and sufficient that $Q$ is in the "double commutant" ([3, Definition 7.1.1]) of $T$. If $S \in B L(X, X)$ is arbitrary, there is the implication

$$
S T=T S \Longrightarrow Q S=S Q \text {. }
$$

In infinite dimensions the situation is more complicated, and it is the purpose of this discussion to classify various relationships between the projection $Q$ and the operator $T$ according to various kinds of disjointness between the spectra of the reduced operators. The discussion can be extended to Banach algebra elements, although it is helpful if the algebras behave to some degree like algebras of operators; the conditions are that they should be "prime", or "ultraprime". Specifically (6] Definition 5]) an algebra $G$ is prime if there is the implication

$$
L_{T} R_{S}=0 \Longrightarrow T=0 \text { or } S=0,
$$

and ultraprime if there is $k>0$ for which

$$
\left\|L_{T} R_{S}\right\| \geq k\|T\|\|S\| \text {. }
$$

This is satisfied if in particular $G=B L(X, X)$ is the bounded operators on a Banach space.

If $Q=Q^{2} \in G$ is an idempotent in a complex Banach algebra $G$, with an identity $I$, then we can represent elements of $G$ as $2 \times 2$ "operator matrices", writing

$$
G=\left(\begin{array}{cc}
A & M \\
N & B
\end{array}\right)=\left\{\left(\begin{array}{cc}
a & m \\
n & b
\end{array}\right): a \in A, m \in M, n \in N, b \in B\right\}
$$

with

$$
I=\left(\begin{array}{ll}
1 & 0 \\
0 & 1
\end{array}\right) \text { and } Q=\left(\begin{array}{ll}
1 & 0 \\
0 & 0
\end{array}\right)
$$

so that $A$ and $B$ are Banach algebras and $M$ and $N$ are bimodules over $A$ and $B$. An element $T \in G$ commuting with $Q$ can be written as

$$
T=\left(\begin{array}{ll}
a & 0 \\
0 & b
\end{array}\right) \in\left(\begin{array}{cc}
A & 0 \\
0 & B
\end{array}\right)=\operatorname{comm}_{G}(Q) .
$$

The partial spectra $\sigma\left(T_{1}\right)$ and $\sigma\left(T_{0}\right)$ are given by the spectra $\sigma_{A}(a)$ and $\sigma_{B}(b)$ relative to the "corner" algebras $A \cong Q G Q$ and $B \cong(I-Q) G(I-Q)$. The condition that $Q$ be in the double commutant of $T$ turns out to be weaker than the disjointness of the spectra $\sigma(a)$ and $\sigma(b)$ : we shall see that it lies somewhere between disjointness of "point spectra" $\pi(a)$ and $\pi(b)$ and disjointness of "approximate point spectra" $\tau(a)$ and $\tau(b)$. We recall ([3, Definition 9.2.1]) that for $T \in G$ the left and right point spectrum $\pi_{G}^{\text {left }}(T)$ and $\pi_{G}^{\text {right }}(T)$ are the eigenvalues of the left and right multiplication operators $L_{T}: G \rightarrow G$ and $R_{T}: G \rightarrow G$, while the left 
and right approximate point spectrum $\tau_{G}^{\text {left }}(T)$ and $\tau_{G}^{\text {right }}(T)$ are the approximate eigenvalues of the same operators. Naturally all these are subsets of the spectrum $\sigma_{G}(T)=\left\{\lambda \in \mathbf{C}: T-\lambda I \notin G^{-1}\right\}$. More generally we need point and approximate point spectra for systems of Banach algebra elements $T=\left(T_{1}, T_{2}, \ldots, T_{n}\right) \in G^{n}$ (3, Definition 11.1.2]):

$$
\pi_{G}^{l e f t}(T)=\left\{\lambda \in \mathbf{C}^{n}: \bigcap_{j=1}^{n}\left(L_{T_{j}}-\lambda_{j} I\right)^{-1}(0) \neq\{0\}\right\}
$$

and

$$
\pi_{G}^{r i g h t}(T)=\left\{\lambda \in \mathbf{C}^{n}: \bigcap_{j=1}^{n}\left(R_{T_{j}}-\lambda_{j} I\right)^{-1}(0) \neq\{0\}\right\} .
$$

Also the left and the right approximate point spectrum of $T$ in $G$ are the simultaneous approximate eigenvalues of the same operators

$$
\tau_{G}^{l e f t}(T)=\left\{\lambda \in \mathbf{C}^{n}: \inf _{\|U\| \geq 1} \sum_{j=1}^{n}\left\|\left(T_{j}-\lambda_{j} I\right) U\right\|=0\right\}
$$

and

$$
\tau_{G}^{r i g h t}(T)=\left\{\lambda \in \mathbf{C}^{n}: \inf _{\|U\| \geq 1} \sum_{j=1}^{n}\left\|U\left(T_{j}-\lambda_{j} I\right)\right\|=0\right\} .
$$

The one way spectral mapping theorem (3 Theorem 11.2.2]) says that there is inclusion, for each $\omega=\pi^{l e f t}, \pi^{\text {right }}, \tau^{\text {left }}, \tau^{\text {right }}$,

$$
f \omega_{G}(T) \subseteq \omega_{G} f(T),
$$

for $m$-tuples of non-commutative polynomials $f: G^{n} \rightarrow G^{m}$, with equality ([3], Theorem 11.3.4]) when $\omega=\tau^{l e f t}, \tau^{\text {right }}$ for commuting systems $T \in G^{n}$. In particular, in the situation of $(0.2)$, we look at pairs

$$
\left(L_{a}, R_{b}\right) \in E^{2}=B L(M, M)^{2},\left(R_{a}, L_{b}\right) \in F^{2}=B L(N, N)^{2} .
$$

The one way spectral mapping theorem gives inclusion

$$
\pi_{E}^{\text {left }}\left(L_{a}, R_{b}\right) \subseteq \pi_{A}^{\text {left }}(a) \times \pi_{B}^{r i g h t}(b) \text { and } \pi_{F}^{l e f t}\left(R_{a}, L_{b}\right) \subseteq \pi_{A}^{\text {right }}(a) \times \pi_{B}^{\text {left }}(b),
$$

and also

$$
(0.5) \tau_{E}^{\text {left }}\left(L_{a}, R_{b}\right) \subseteq \tau_{A}^{\text {left }}(a) \times \tau_{B}^{\text {right }}(b) \text { and } \tau_{F}^{\text {left }}\left(R_{a}, L_{b}\right) \subseteq \tau_{A}^{\text {right }}(a) \times \tau_{B}^{\text {left }}(b) .
$$

Further, if the algebra $G$ is prime then there is equality in (0.4), and if the algebra $G$ is ultraprime there is equality in (0.5). Remark that if the algebra $G=\left(\begin{array}{cc}A & M \\ N & B\end{array}\right)$ is prime, then the analagous condition holds between $a \in A$ and $b \in B$ acting on the spaces $M$ and $N$

$$
L_{a} R_{b}=0 \text { or } R_{a} L_{b}=0 \Longrightarrow a=0 \text { or } b=0
$$

for notice

$$
\begin{aligned}
& \left(\begin{array}{ll}
a & 0 \\
0 & 0
\end{array}\right)\left(\begin{array}{ll}
a^{\prime} & m^{\prime} \\
n^{\prime} & b^{\prime}
\end{array}\right)\left(\begin{array}{ll}
0 & 0 \\
0 & b
\end{array}\right)=\left(\begin{array}{cc}
0 & a m^{\prime} b \\
0 & 0
\end{array}\right) \\
& \left(\begin{array}{ll}
0 & 0 \\
0 & b
\end{array}\right)\left(\begin{array}{cc}
a^{\prime} & m^{\prime} \\
n^{\prime} & b^{\prime}
\end{array}\right)\left(\begin{array}{ll}
a & 0 \\
0 & 0
\end{array}\right)=\left(\begin{array}{cc}
0 & 0 \\
b n^{\prime} a & 0
\end{array}\right)
\end{aligned}
$$


In effect if the algebra $G$ is prime, then so is the induced category of four "objects" $\{A, M, N, B\}$. In the same way ultraprimeness for $G$ transfers to ultraprimeness for $\{A, M, N, B\}$.

In this generality the disjointness of partial spectra $\sigma(a)$ and $\sigma(b)$ says something stronger than double commuting about the projection $Q$ :

1. Theorem. $a \in A$ and $b \in B$ have disjoint spectra

$$
\sigma_{A}(a) \cap \sigma_{B}(b)=\emptyset
$$

if and only if $Q$ is a holomorphic function of $T$ :

$$
\left(\begin{array}{ll}
1 & 0 \\
0 & 0
\end{array}\right) \in \text { Holo }\left(\begin{array}{ll}
a & 0 \\
0 & b
\end{array}\right) \text {. }
$$

Proof. Here we write $Q \in \operatorname{Holo}(T)$ to mean that $Q=f(T)$ with $f$ holomorphic on some open neighbourhood of the spectrum $\sigma(T)$, where $f(T)$ is given by the holomorphic functional calculus ([3, Definition 9.7.1]) based on the Cauchy integral formula. If (1.2) holds, then $f(a)=1$ and $f(b)=0$ so that $\sigma(a) \subseteq f^{-1}(1)$ and $\sigma(b) \subseteq f^{-1}(0)$ lie in disjoint sets, which gives (1.1). Conversely if (1.1) holds, then so does (1.2), with $f=\chi_{K}$ the characteristic function of the spectral set $K=\sigma(a) \subseteq \sigma(a) \cup \sigma(b)=\sigma(T)$.

It is clear from the functional calculus that holomorphic functions of a Banach algebra element lie in the double commutant, so that we can expect the double commutant condition to be somewhat weaker than the disjointness of the full spectra.

2. Theorem. If $a \in A$ and $b \in B$ have disjoint approximate point spectra, in the sense that

$$
\tau_{A}^{l e f t}(a) \cap \tau_{B}^{\text {right }}(b)=\emptyset=\tau_{A}^{r i g h t}(a) \cap \tau_{B}^{l e f t}(b),
$$

then $Q$ is in the double commutant of $T$ :

$$
\left(\begin{array}{ll}
1 & 0 \\
0 & 0
\end{array}\right) \in \operatorname{comm}_{G}^{2}\left(\begin{array}{ll}
a & 0 \\
0 & b
\end{array}\right)
$$

If in particular the algebra $G$ is prime, then conversely this implies that point spectra are disjoint:

$$
\pi_{A}^{l e f t}(a) \cap \pi_{B}^{r i g h t}(b)=\emptyset=\pi_{A}^{r i g h t}(a) \cap \pi_{B}^{l e f t}(b) .
$$

Proof. If (2.2) holds, then

$$
\operatorname{comm}_{G}\left(\begin{array}{cc}
a & 0 \\
0 & b
\end{array}\right) \subseteq \operatorname{comm}_{G}\left(\begin{array}{ll}
1 & 0 \\
0 & 0
\end{array}\right)=\left(\begin{array}{cc}
A & 0 \\
0 & B
\end{array}\right) \subseteq G .
$$

Now from (2.4) follow the implications

$$
\left(\begin{array}{ll}
0 & u \\
0 & 0
\end{array}\right) \in \operatorname{comm}_{G}\left(\begin{array}{ll}
a & 0 \\
0 & b
\end{array}\right) \Longrightarrow u=0 \text { and }\left(\begin{array}{ll}
0 & 0 \\
v & 0
\end{array}\right) \in \operatorname{comm}_{G}\left(\begin{array}{ll}
a & 0 \\
0 & b
\end{array}\right) \Longrightarrow v=0 .
$$

In words the operators $L_{a}-R_{b}: M \rightarrow M$ and $R_{a}-L_{b}: N \rightarrow N$ are one-to-one:

$$
0 \notin \pi_{E}^{l e f t}\left(L_{a}-R_{b}\right) \text { and } 0 \notin \pi_{F}^{l e f t}\left(R_{a}-L_{b}\right) .
$$

Now the one way spectral mapping theorem (0.3) says

$$
\begin{gathered}
\left\{\lambda-\mu:(\lambda, \mu) \in \pi_{E}^{\text {left }}\left(L_{a}, R_{b}\right)\right\} \subseteq \pi_{E}^{\text {left }}\left(L_{a}-R_{b}\right) \text { and } \\
\left\{\lambda-\mu:(\lambda, \mu) \in \pi_{F}^{l e f t}\left(R_{a}, L_{b}\right)\right\} \subseteq \pi_{F}^{l e f t}\left(R_{a}-L_{b}\right),
\end{gathered}
$$


and this with the prime condition (equality in (0.4)) gives disjointness (2.3). Conversely the condition (2.5), and a fortiori the stronger condition

$$
0 \notin \tau_{E}^{l e f t}\left(L_{a}-R_{b}\right) \text { and } 0 \notin \tau_{F}^{l e f t}\left(R_{a}-L_{b}\right),
$$

is sufficient for the double commutivity (2.4), and then (2.7) follows from (2.1). Neither primeness nor ultraprimeness is needed at this stage, but we do need the two-way spectral mapping theorem for $\tau$ so that there is equality in $(0.3)$ :

$$
\begin{gathered}
\left\{\lambda-\mu:(\lambda, \mu) \in \tau_{E}^{\text {left }}\left(L_{a}, R_{b}\right)\right\}=\tau_{E}^{\text {left }}\left(L_{a}-R_{b}\right) \text { and } \\
\left\{\lambda-\mu:(\lambda, \mu) \in \tau_{F}^{\text {left }}\left(R_{a}, L_{b}\right)\right\}=\tau_{F}^{\text {left }}\left(R_{a}-L_{b}\right) .
\end{gathered}
$$

The condition (2.5) is equivalent to the condition (2.4) and hence also (2.2), but possibly stronger than the disjointness (2.3): suppose for example $b=a$ with $\pi^{l e f t}(a)=\emptyset$. A specific example would be the forward shift. When the algebra $G$ is ultraprime however the conditions (2.1) and (2.7) are the same. A feeble intimation of the disjointness (1.1) follows (cf. 6. (11.2)]) from the disjointness of the point spectra, and hence from the double commutant condition (2.2):

$$
\begin{aligned}
\sigma_{A}^{\text {left }}(a) \cap \sigma_{B}^{\text {right }}(b) \subseteq \sigma_{A}^{\text {right }}(a) \cup \sigma_{B}^{\text {left }}(b) \text { and } \\
\\
\quad \sigma_{A}^{\text {right }}(a) \cap \sigma_{B}^{\text {left }}(b) \subseteq \sigma_{A}^{\text {left }}(a) \cup \sigma_{B}^{\text {right }}(b) .
\end{aligned}
$$

Even in finite dimensions the double commutant condition (2.2), and hence also the disjointness condition (1.1), need not hold (8 Example 3.1). Provided the disjointness condition (1.1) is satisfied, the idempotent $Q=\left(\begin{array}{ll}1 & 0 \\ 0 & 0\end{array}\right)$ of (1.2) and (2.2) is the only projection commuting with the element $T=\left(\begin{array}{ll}a & 0 \\ 0 & b\end{array}\right) \in G$ which induces the correct spectra for the elements $a \in A$ and $b \in B$ : for if $P$ is another, then by a joint spectrum argument (5 (6.7)) the spectrum of each of the projections $Q-Q P$ and $P-Q P$ reduces to $\{0\}$. The arguments that (1.2) implies (1.1) and that (2.1) implies (2.2) are due to Koliha [8, in the finite dimensional case, where of course (1.1) and (2.3) are equivalent. Implication $(2.1) \Longrightarrow(2.2)$ is also a consequence of Rosenblum's theorem ([10, Corollary 0.14]). For matrices we can actually deploy the Euclidean algorithm to show [7, [9] that the idempotent $\left(\begin{array}{ll}1 & 0 \\ 0 & 0\end{array}\right)$ is a polynomial function of the operator $\left(\begin{array}{ll}a & 0 \\ 0 & b\end{array}\right)$. Also in the finite dimensional case the equivalence between (1.2) and (2.2) is a deep theorem (cf. [8, Theorem 5.1], [13, page 106], [12, Theorem 1.7.3]) which does not however survive in infinite dimensions.

3. Example. If $A=B=M=N=B L(X, X)$ is the algebra of bounded operators on the sequence space $X=\ell_{2}$ and if $u \in A$ is the forward shift $\left(\xi_{1}, \xi_{2}, \xi_{3}, \ldots\right) \mapsto$ $\left(0, \xi_{1}, \xi_{2}, \ldots\right)$, then

$$
\left(\begin{array}{ll}
1 & 0 \\
0 & 0
\end{array}\right) \in \operatorname{comm}^{2}\left(\begin{array}{cc}
u & 0 \\
0 & 1-u
\end{array}\right)
$$

but

$$
\left(\begin{array}{ll}
1 & 0 \\
0 & 0
\end{array}\right) \notin \text { Holo }\left(\begin{array}{cc}
u & 0 \\
0 & 1-u
\end{array}\right)
$$

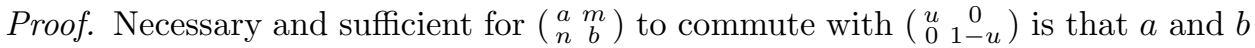
commute with $u$ while $x=m$ and $x=n$ satisfy the equation

$$
u x+x u=x .
$$


Since the operator $I-L_{u}-R_{u}$ is one-to-one this forces $m=n=0$. To see this recall the backward shift $v \in A$, so that $v u=1 \neq u v$ : now if $u x+x u=x$ and $w \in A$ is arbitrary we claim that there is implication

$$
x=w x \Longrightarrow(1-u w v) x(1-u)=0 \Longrightarrow x=u w v x .
$$

The first implication here is elementary algebra, and the second follows because $1-u \in A$ has dense range. By induction, starting with $w=1$, it follows that if $u x+x u=x$, then

$$
x=u^{n} v^{n} x \in u^{n} A \text { for each } n \in \mathbf{N},
$$

so forcing $x=0$. Thus we have (3.1): for (3.2) observe

$$
1 \in \sigma(u) \cap \sigma(1-u) \neq \emptyset
$$

and recall Theorem 1.

If we write $T=\left(\begin{array}{ll}a & 0 \\ 0 & b\end{array}\right)$ as in (0.2) and define $\mathbf{P}: G \rightarrow G$ by setting

$$
\mathbf{P}\left(\left(\begin{array}{cc}
a^{\prime} & m^{\prime} \\
n^{\prime} & b^{\prime}
\end{array}\right)\right)=\left(\begin{array}{cc}
a^{\prime} & 0 \\
0 & b^{\prime}
\end{array}\right)
$$

then the commutant condition (2.4) can be written in the form

$$
\left(L_{T}-R_{T}\right)^{-1}(0) \subseteq \mathbf{P}(G),
$$

which is of course equivalent to the spectral condition (2.5). The stronger spectral condition (2.7) should therefore be equivalent to a strengthened version (3. Definition 10.3.1]; cf. [3. (9.5)]) of the "exactness" (3.5):

4. Theorem. With the notation (3.4), necessary and sufficient for the boundedness below (2.7) is that there are $k>0$ and $h>0$ for which: for arbitrary $U \in G$ there is $\left(V_{n}\right)$ in $G$ for which

$$
\underset{n}{\limsup }\left\|U-\mathbf{P}\left(V_{n}\right)\right\| \leq k\left\|\left(L_{T}-R_{T}\right)(U)\right\| \text { with } \sup _{n}\left\|V_{n}\right\| \leq h\|U\| .
$$

Proof. We claim that (2.7) is equivalent to the following "comparison" (cf. [3, Definition 10.1.1]): there is $k>0$ for which

$$
\|(\mathbf{I}-\mathbf{P})(\cdot)\| \leq k\left\|\left(L_{T}-R_{T}\right)(\cdot)\right\| \text { on } G .
$$

To see this observe, for arbitrary $a^{\prime}, m^{\prime}, n^{\prime}, b^{\prime}$,

$$
\left\|\left(\begin{array}{cc}
0 & m^{\prime} \\
n^{\prime} & 0
\end{array}\right)\right\| \leq k\left\|\left(\begin{array}{cc}
0 & a m^{\prime}-m^{\prime} b \\
b n^{\prime}-n^{\prime} a & 0
\end{array}\right)\right\| \leq k\left\|\left(\begin{array}{cc}
a a^{\prime}-a^{\prime} a & a m^{\prime}-m^{\prime} b \\
b n^{\prime}-n^{\prime} a & b b^{\prime}-b^{\prime} b
\end{array}\right)\right\| .
$$

This proves that the boundedness below (2.7) is sufficient for the operator majorization (4.2), taking the norm of an operator matrix to be the maximum of the norms of its entries. Conversely apply $L_{T}-R_{T}$ to $\left(\begin{array}{cc}0 & m^{\prime} \\ 0 & 0\end{array}\right)$ and to $\left(\begin{array}{cc}0 & 0 \\ n^{\prime} & 0\end{array}\right)$. It is clear that (4.2) is sufficient for (4.1): take $V_{n}=U$ for each $n \in \mathbf{N}$. Conversely if (4.1) holds, then premultiply by $\mathbf{P}$ and subtract to obtain (4.2), with $(1+\|\mathbf{P}\|) k$ in place of $k$.

Even stronger than (4.1) is "split exactness": 
5. Theorem. With the notation of (3.4), disjointness

$$
\sigma_{A}^{l e f t}(a) \cap \sigma_{B}^{r i g h t}(b)=\emptyset=\sigma_{A}^{r i g h t}(a) \cap \sigma_{B}^{l e f t}(b)
$$

implies that there are $\mathbf{U}$ and $\mathbf{V}$ on $G$ for which

$$
\mathbf{V}\left(L_{T}-R_{T}\right)+\mathbf{P U}=\mathbf{I},
$$

which in turn, provided $G$ is ultraprime, implies disjointness (2.1).

Proof. We prove that left invertibility

$$
U\left(L_{a}-R_{b}\right)=I \in E \text { and } V\left(L_{b}-R_{a}\right)=I \in F
$$

is equivalent to the factorization

$$
\mathbf{I}-\mathbf{P}=\mathbf{W}\left(L_{T}-R_{T}\right):
$$

certainly if (5.3) holds, then so does (5.4), with

$$
\mathbf{W}\left(\begin{array}{cc}
a^{\prime} & m^{\prime} \\
n^{\prime} & b^{\prime}
\end{array}\right)=\left(\begin{array}{cc}
0 & U m^{\prime} \\
V n^{\prime} & 0
\end{array}\right) .
$$

Conversely if (5.4) holds we can extract $U \in E$ and $V \in F$ from

$$
\left(\begin{array}{cc}
* & U m^{\prime} \\
* & *
\end{array}\right)=\mathbf{W}\left(\begin{array}{cc}
0 & m^{\prime} \\
0 & 0
\end{array}\right) \text { and }\left(\begin{array}{cc}
* & * \\
V n^{\prime} & *
\end{array}\right)=\mathbf{W}\left(\begin{array}{cc}
0 & 0 \\
n^{\prime} & 0
\end{array}\right) \text {. }
$$

It is clear that spectral disjointness (5.1) is sufficient for left invertibility (5.3), and that the factorization (5.4) is stronger than the majorization (4.2). Majorization (4.2) is equivalent to boundedness below (2.7), which if $G$ is ultraprime implies disjointness (2.1).

Spectral disjointness (5.1) says something about the spectra of triangular matrices extending $\left(\begin{array}{ll}a & 0 \\ 0 & b\end{array}\right)[1],[2]$ :

6. Theorem. If $a \in A, b \in B$ and $m \in M$ are arbitrary, then there is inclusion

$$
\begin{aligned}
\sigma_{A}^{\text {left }}(a) \cup \sigma_{B}^{r i g h t}(b) & \subseteq \sigma_{G}\left(\begin{array}{cc}
a & m \\
0 & b
\end{array}\right) \subseteq \sigma_{A}(a) \cup \sigma_{B}(b) \\
& \subseteq \sigma_{G}\left(\begin{array}{cc}
a & m \\
0 & b
\end{array}\right) \cup\left(\sigma_{A}^{\text {right }}(a) \cap \sigma_{B}^{\text {left }}(b)\right),
\end{aligned}
$$

with

$$
\partial\left(\sigma_{A}(a) \cup \sigma_{B}(b)\right) \subseteq \sigma_{G}\left(\begin{array}{cc}
a & m \\
0 & b
\end{array}\right)
$$

If $a \in A$ and $b \in B$ are arbitrary, then the following are equivalent:

$$
\left(\begin{array}{cc}
a & m \\
0 & b
\end{array}\right) \in G^{-1} \text { is invertible for some } m \in M
$$

there are $a^{\prime} \in A$ and $b^{\prime} \in B$ for which

$$
a^{\prime} a=1 \in A \text { and } b b^{\prime}=1 \in B
$$

with similarity of complementary idempotents

$$
1-a a^{\prime} \sim 1-b^{\prime} b .
$$


Proof. This is very elementary. We observe (4, Theorem 3.1])

$$
\begin{gathered}
\sigma_{A}^{\text {left }}(a) \subseteq \sigma_{G}^{\text {left }}\left(\begin{array}{cc}
a & m \\
0 & b
\end{array}\right) \subseteq \sigma_{A}^{\text {left }}(a) \cup \sigma_{B}^{\text {left }}(b), \\
\sigma_{B}^{\text {right }}(b) \subseteq \sigma_{G}^{\text {right }}\left(\begin{array}{cc}
a & m \\
0 & b
\end{array}\right) \subseteq \sigma_{A}^{\text {right }}(a) \cup \sigma_{B}^{\text {right }}(b)
\end{gathered}
$$

and ([4 Theorem 3.2])

$$
\sigma_{A}^{r i g h t}(a) \subseteq \sigma_{G}^{r i g h t}\left(\begin{array}{cc}
a & m \\
0 & b
\end{array}\right) \cup \sigma_{B}^{\text {left }}(b)
$$

and

$$
\sigma_{B}^{l e f t}(b) \subseteq \sigma_{G}^{l e f t}\left(\begin{array}{cc}
a & m \\
0 & b
\end{array}\right) \cup \sigma_{A}^{r i g h t}(a) .
$$

This gives (6.1). Then for (6.2) we argue ([3, Theorems 7.10.3, 9.3.3])

$$
\partial\left(\sigma_{A}(a) \cup \sigma_{B}(b)\right) \subseteq \partial \sigma_{A}(a) \cup \partial \sigma_{B}(b) \subseteq \sigma_{A}^{l e f t}(a) \cup \sigma_{B}^{r i g h t}(b) .
$$

If (6.3), then $\left(\begin{array}{cc}a & m \\ 0 & b\end{array}\right)$ has a two-sided inverse $\left(\begin{array}{cc}a^{\prime} & \begin{array}{c}m^{\prime} \\ n^{\prime}\end{array} \\ b^{\prime}\end{array}\right) \in\left(\begin{array}{cc}A & M \\ N & B\end{array}\right)$, so that matrix multiplication gives (6.4) and

$$
1-a a^{\prime}=m n^{\prime} \in A \text { and } 1-b^{\prime} b=n^{\prime} m,
$$

which is the similarity (6.5). Conversely if (6.4) and (6.6) hold, then $1-b^{\prime} b$ and $1-a a^{\prime}$ are idempotents, and (6.6) continues to hold with $\left(1-b^{\prime} b\right) n^{\prime}\left(1-a a^{\prime}\right)$ in place of $n^{\prime}$. Thus without loss of generality we may assume

$$
n^{\prime}=\left(1-b^{\prime} b\right) n^{\prime}=n^{\prime}\left(1-a a^{\prime}\right)
$$

so that

$$
b n^{\prime}=0=n^{\prime} a
$$

and hence

$$
\left(\begin{array}{cc}
a^{\prime} & 0 \\
n^{\prime} & b^{\prime}
\end{array}\right)\left(\begin{array}{cc}
a & m \\
0 & b
\end{array}\right)=\left(\begin{array}{cc}
1 & a^{\prime} m \\
0 & 1
\end{array}\right) \text { and }\left(\begin{array}{cc}
a & m \\
0 & b
\end{array}\right)\left(\begin{array}{cc}
a^{\prime} & 0 \\
n^{\prime} & b^{\prime}
\end{array}\right)=\left(\begin{array}{cc}
1 & m b^{\prime} \\
0 & 1
\end{array}\right)
$$

are both invertible, giving (6.3).

From (6.1) it follows (cf. [10, Corollary 0.15]) that

$$
\sigma_{A}^{r i g h t}(a) \cap \sigma_{B}^{l e f t}(b)=\emptyset \Longrightarrow \sigma_{G}\left(\begin{array}{cc}
a & m \\
0 & b
\end{array}\right)=\sigma_{G}\left(\begin{array}{cc}
a & 0 \\
0 & b
\end{array}\right) .
$$

The spectral theory argument ([6] Lemma 3 , Theorem 4]) says that the "co approximate point spectrum" or defect spectrum of the multiplication operator $L_{a}-R_{b}$ on $M$ is given by the algebraic difference between the right and left spectra of $a$ and $b$ :

$$
\tau_{E}^{\text {right }}\left(L_{a}-R_{b}\right)=\sigma_{A}^{\text {right }}(a)-\sigma_{B}^{\text {left }}(b) .
$$

If their intersection is empty it follows that $L_{a}-R_{b}: M \rightarrow M$ is onto, and hence that the upper triangular $\left(\begin{array}{ll}a & m \\ 0 & b\end{array}\right)$ is similar to the diagonal $\left(\begin{array}{ll}a & 0 \\ 0 & b\end{array}\right)$. We take the opportunity to point out a misprint in the statement of Theorem 4 of [6]: on the right hand sides of the second parts of [6] (4.2) and (4.3) the $\tau$ and the $\pi$ should be $\sigma$. 
We conclude with a slight extension of Wermuth's theorem [14 about commuting exponentials, adapting the argument of Schmoeger [11]:

7. Theorem. If the left and right approximate point spectra of $a \in A$ and $b \in B$ are "mutually $2 \pi i$-congruence-free", in the sense that

$$
\lambda \in \tau_{A}^{l e f t}(a), \mu \in \tau_{B}^{r i g h t}(b), e^{\lambda}=e^{\mu} \Longrightarrow \lambda=\mu,
$$

then there is equality

$$
\left(L_{e^{a}}-R_{e^{b}}\right)^{-1}(0)=\left(L_{a}-R_{b}\right)^{-1}(0) \subseteq M .
$$

If in particular (7.1) holds with $A=B$ and $a=b=c$ and also with $a=b=c^{\prime}$, then there is the implication

$$
e^{c^{\prime}} e^{c}=e^{c} e^{c^{\prime}} \Longrightarrow c^{\prime} c=c c^{\prime} .
$$

Proof. Recall, following Schmoeger 11], the "difference quotient" $\delta_{\alpha} f$ of a holomorphic function $f: U \rightarrow \mathbf{C}$ at a point $\alpha \in U$ :

$$
\left(\delta_{\alpha} f\right)(\lambda)=\frac{f(\lambda)-f(\alpha)}{\lambda-\alpha} \text { if } \lambda \neq \alpha,=f^{\prime}(\alpha) \text { if } \lambda=\alpha .
$$

Evidently $\delta_{\alpha} f: U \rightarrow \mathbf{C}$ is holomorphic on the same set as $f$. When in particular $f=\exp$ is the exponential function notice that if $\lambda \neq \mu$ are distinct points of $\mathbf{C}$ there is the equivalence

$$
e^{\lambda}=e^{\mu} \Longleftrightarrow\left(\delta_{0} \exp \right)(\lambda-\mu)=0
$$

Now the formula (7.4) says that, with $\varphi=\delta_{0} \exp$,

$$
R_{e^{b}} \varphi\left(L_{a}-R_{b}\right)\left(L_{a}-R_{b}\right)=\left(L_{e^{a}}-R_{e^{b}}\right) .
$$

By (2.8) and (0.3) we have inclusion

$$
\pi_{E}^{l e f t} \varphi\left(L_{a}-R_{b}\right) \subseteq \tau_{E}^{l e f t} \varphi\left(L_{a}-R_{b}\right)=\varphi \tau_{E}^{l e f t}\left(L_{a}-R_{b}\right) \subseteq \varphi\left(\tau_{A}^{l e f t}(a)-\tau_{B}^{\text {right }}(b)\right) .
$$

Thus the condition (7.1), together with the equivalence (7.5), ensures that the operator $\varphi\left(L_{a}-R_{b}\right)$ is bounded below, in particular one-to-one. This in the formula (7.6) gives the inclusion (7.2). Specialising to $A=B=M$ and $a=b=c$ gives the inclusion

$$
\operatorname{comm}_{A}\left(e^{c}\right) \subseteq \operatorname{comm}_{A}(c) \subseteq A .
$$

In particular there is implication, for arbitrary $c^{\prime} \in A$,

$$
e^{c^{\prime}} e^{c}=e^{c} e^{c^{\prime}} \Longrightarrow e^{c^{\prime}} c=c e^{c^{\prime}}
$$

but if also the condition (7.1) holds with $a=b=c^{\prime}$ there is also the implication

$$
e^{c^{\prime}} c=c e^{c^{\prime}} \Longrightarrow c^{\prime} c=c c^{\prime} \text {. }
$$

\section{REFERENCES}

1. Hong-Ke Du and Jin Pan, Perturbation of spectrums of $2 \times 2$ operator matrices, Proc. Amer. Math. Soc. 121 (1994) 761-766. MR 94i:47004

2. Jin Kyu Han, Hong Youl Lee and Woo Young Lee, Invertible completions of $2 \times 2$ upper triangular operator matrices, Proc. Amer. Math. Soc. 128 (2000) 119-123. MR 2000c:47003

3. R.E. Harte, Invertibility and singularity, Dekker New York 1988. MR 89d:47001

4. R.E. Harte, Invertibility and singularity for operator matrices, Proc. Royal Irish Acad. 88A (1988) 103-118. MR 90a:15019

5. R.E. Harte, Unspectral sets, Rendiconti del Circ. Mat. Palermo 56 (1998) 69-77. CMP 2000:01 
6. R.E. Harte and C. Hernandez, On the Taylor spectrum of left-right multipliers, Proc. Amer. Math. Soc. 126 (1998) 397-404. MR 98e:46057

7. K. Hoffman and R. Kunze, Linear algebra, Prentice Hall New York 1971. MR 43:1998

8. J.J. Koliha, Block diagonalization, Mathematica Bohemica (to appear).

9. R.R. London and H.P. Rogosinski, Decomposition theory in the teaching of elementary linear algebra, Amer. Math. Monthly 97 (1990) 478-485. MR 91d:15022

10. H. Radjavi and P. Rosenthal, Invariant subspaces, Springer New York 1973. MR 51:3924

11. Ch. Schmoeger, Remarks on commuting exponentials on Banach algebras, Proc. Amer. Math. Soc. 127 (1999) 1337-1338. MR 99h:46090

12. D. A. Suprunenko and R.I. Tyshkevich, Commutative matrices, Academic Press New York 1968.

13. J. H. M. Wedderburn, Lectures on matrices, AMS Colloq. Publ. 17, Amer. Math. Soc. Providence 1934.

14. E. M. E. Wermuth, A remark on commuting operator exponentials, Proc. Amer. Math. Soc. 125 (1997) 1685-1688. MR 97g:39011

School of Mathematics, Trinity College, Dublin 2, Ireland

E-mail address: rharte@maths.tcd.ie 